\title{
Building Conceptual Dictionaries for an Integrated Narrative Generation System
}

\author{
Takashi Ogata \\ Faculty of Software and Information Science, Iwate Prefectural University, Takizawa, Iwate, Japan \\ t-ogata@iwate-pu.ac.jp
}

\begin{abstract}
Integrated Narrative Generation System (INGS) is a framework for integrating our previous researches on narrative generation. INGS integrates various narrative generation modules into an organic architecture. This paper presents a verb conceptual dictionary and a noun conceptual dictionary to be used in INGS. Each of the dictionaries has a conceptual hierarchy with single inheritance by is-a relations. A terminal concept in the verb conceptual dictionary describes one or more case frame(s), the constraints of the main elements and a sentence pattern. The basic description of each terminal concept in the noun conceptual dictionary associates the concept to the other noun concept. The purpose of the study is not developing general conceptual dictionaries, but building them as a mechanism in narrative generation, especially in INGS. Actually, we have combined the two developed conceptual dictionaries with INGS. In this paper, we will present the basic mechanisms based on the cooperation of two types of conceptual dictionary through an implemented narrative generation system, INGS.
\end{abstract}

Keywords: Narrative generation, Integrated Narrative Generation System (INGS), Noun conceptual dictionary, Verb conceptual dictionary, Case frame, Constraint.

\section{Introduction}

Narrative generation or story generation as one of the difficult research issues in artificial intelligence and cognitive science aims at generating narratives or stories automatically by computer. Many systems have been proposed in the field. An approach is by planning and goal-plan strategy [1]. TALE-SPIN [2] is the pilot program and automatically generates different story texts according to characters' planning actions toward achieving specific goals. DAYDREAMER [3] and MINSTREL [4] have more comprehensive and complicated architectures based on the above methods. The story generation system based on an Aesop world [5] uses a goal-plan mechanism, as well as large-scaled conceptual dictionaries to make using story representation. Another main approach is grounded on story grammar and story schema for narrative structural formalization. GESTER [6] and Joseph [7], for example, respectively consist of an interpreter with both a set of story grammar and a world model. On the other hand, BRUTUS [8] shows a blended approach of planning and structural methods. There is also third approach for interactive drama or interactive story telling as an application of narrative generation. Oz project [9] developed an interactive drama with dialogue and actions in autonomous agents and Monfort [10] proposed an interactive storytelling system using a mechanism for controlling narrative viewpoints.

We have also been conducting on research of developing narrative generation systems as intelligent tools, in various meanings, for producing and creating digital narratives and further literary texts [11]. One of the main current research topics is to design and develop the Integrated Narrative Generation System (INGS) [12,13]. INGS integrates diverse narrative elements into an organically integrated architecture based on a variety of background conceptual ideas or concepts relating to narrative generation, such as "expanded literary theory" [14,15] and "circulative narrative generation" [16]. The expanded literary theory is the conception of a new literary theory, on which existing literary theories and narratology are used in the reconstruction for informatics. INGS actually applies several literary theories to the important functions or 
elements as in the next section. On the other hand, the concept of circulative narrative generation is implemented in INGS as functions including generating based on the mutual repetition between story generation and music generation [17].

The eventual goal of INGS is a versatile narrative generation. We mean by the "versatile" the potential to be used and applied to various purposes relevant to narrative generation. The above BRUTUS system [8] deals with only a specialized narrative theme, "betrayal", while INGS intends to develop a more general mechanism for various types of narratives based on a versatile characteristic grounded on an integrated architecture. Various intentional revisions of the structural techniques and narrative contents knowledge in INGS will enable the transformation of the genre and theme. Comparatively large-scaled conceptual dictionaries are also mechanisms for the versatile and general narrative generation because of no having the limitation of previously decided narrative themes and genres.

The objective of this paper is to introduce the present version of two conceptual dictionaries in INGS: a noun conceptual dictionary and a verb conceptual dictionary. These conceptual dictionaries respectively have a hierarchical structure including terminal nodes to be associated with basic or general language concepts, such as "apple", and intermediate nodes for semantically combining the lower nodes including the terminal nodes, such as "fruits". The main role is to give semantic information to an "event concept" that is the most fundamental element in a narrative conceptual structure generated by INGS. Narrative structures generated by INGS in a generation process are divided into conceptual structures and surface structures. The surface structures include structures by language, music, and so on, while the conceptual structures mean internal semantic narrative representation to be expressed by the surface structures. The conceptual structures are divided into a story conceptual structure and a discourse conceptual structure. An event concept or an event simply is corresponded to a basic unit in their narrative conceptual structures. An event concept basically consists of a verb concept and several noun concepts. The concepts are associated with the corresponding elements and descriptions in the conceptual dictionaries. As mentioned above, a group of conceptual dictionaries in INGS is an important knowledge element for providing some types of knowledge to each event concept which is a basic unit in narrative conceptual structures generated by INGS.

As papers relating to the conceptual dictionaries in INGS, we have been presenting the idea and plan [18], the process of implementation [19], and the application to a special issue [20]. This paper is a general overview of the conceptual dictionaries in the present phase of INGS as an explorative and incremental study. Additionally, the basic part of conceptual dictionaries for adjectives and adjective verbs has also been developing and proper noun concepts and attribute frames (using concepts) have been adding to the noun conceptual dictionary. The truly comprehensive systematization of conceptual dictionaries in INGS will be addressed continuously in the future.

The story generation system by Okada and Endo [5], as mentioned above, uses general large-scaled conceptual dictionaries in the story generation mechanism, but is limited to only one narrative theme and one narrative world. In contrast, INGS architecture is constructed towards the versatility and generality of generation through an organic fusion of diverse narrative knowledge and techniques including planning, discourse structure, story grammar, script, discourse relation, and so on. And in contrast with the above Aesop system is positioned as an application of conceptual dictionary research, INGS pursues a comprehensive mechanism of narrative generation itself. To develop conceptual dictionaries themselves is not our extreme purpose. On the other hand, a story generation system by McIntyre and Lapata [21] generates stories by using a knowledge base about compositions of an event sentence and chains of events extracted from a narrative corpus based on co-occurrence of words. Stories are generated by a kind of tree search of possible stories. This knowledge base is not a conceptual dictionary and the system does not use general conceptual dictionaries. In INGS, this kind of knowledge for making chains or sequences of events is defined as a relatively dependent group of knowledge, story content knowledge base. INGS architecture is more comprehensive and modular than many of the other narrative generation architectures.

Table 1. compares existing conceptual dictionaries for the Japanese language. "Goi-Taikei, A Japanese Lexicon” [22] is one of the largest Japanese lexical and conceptual dictionary for Japanese-to-English machine translation, which hierarchically organizes semantic 
attributes according to a single inheritance. We have referred to this at the starting point to construct the overall structures of our conceptual dictionaries. In particular, as a way of refining and elaborating, we have referred to "Japanese WordNet" [23] for the purpose of complementing the shortage of intermediate concepts in the noun conceptual hierarchy to a large degree. In summary, on our noun conceptual dictionary, the basic structure of the higher level basically has been organized referring to the "general semantic attributes system (hierarchy) for nouns" in "Japanese Lexicon”, while the hierarchy of lower intermediate concepts has been defined referring to "Japanese WordNet". And the terminal concepts basically use words in "Japanese Lexicon”. However, these references are limited to the first developmental stage of the conceptual dictionaries. We have been implementing an original organization of the conceptual dictionaries according to the task of our narrative generation. In particular, the specific form of an event which is a basic unit in a generated narrative structure is given by a case frame and the constraints in the verb conceptual dictionary and the content knowledge is provided by the noun conceptual dictionary. The necessity in our narrative generation incrementally will refine and elaborate the structures, organization, and contents of the conceptual dictionaries.

\section{An Overview of INGS}

In the macro design of our research, the architecture of narrative generation consists of conceptual generation phase and surface representation phase. The former is divided into the part of generating a story structure as the narrative content to be narrated and the part of generating a discourse structure as the structural transformation of a story structure. The surface representation media include natural language, visual media, music, and so on. According to this framework, we have been developing various mechanisms and modules independently of each other. INGS synthesizes these existing mechanisms in a same framework. A variety of the modules are organically blended into a whole. The goal of this system is to execute the generation of narrative structures in some levels such as story, discourse and surface representations in a unified method. Fig. 1. shows the INGS architecture. The system is consisted of several types of modules relating to knowledge bases, narrative techniques for narrative structures' generation and transformation, and the control. INGS is mainly implemented by Common Lisp with 865 defined functions currently.

INGS has been developing through an exploratory approach. In particular, we have been employing a blended approach of both the top-down approach relevant to the system's macro design and the bottom-up approach of designing and implementing each of the modules. And through such process, conceptual dictionaries have been playing an important role for an organized synthesis of some modules developed independently. Standardizing the description of an event form and the reference of common conceptual dictionaries from various modules has been contributing to the combination as a whole narrative generation system. This is an important function of the conceptual dictionaries from the viewpoint of the development process of INGS.

Additionally, the "integrated" in INGS has the following meanings: integrations of 1) narrative generation phases, 2) expression media, 3) literary theories and informatics, and 4) diverse techniques for

Table 1. Conceptual Dictionaries for the Japanese Language.

\begin{tabular}{|l|l|l|l|}
\hline & $\begin{array}{l}\text { EDR Electronic Dictionary } \\
\text { National Institute of Information and } \\
\text { Communications Technology, 2001)[24] }\end{array}$ & $\begin{array}{l}\text { Japanese WordNet } \\
\text { [23][25] }\end{array}$ & Goi-Taikei,A Japanese Lexicon[22] \\
\hline $\begin{array}{l}\text { The number } \\
\text { of concepts }\end{array}$ & About 410,000 & 57,238 & About 3,000 \\
\hline $\begin{array}{l}\text { The number } \\
\text { of words }\end{array}$ & About 270,000 & 93,834 & About 300,000 \\
\hline $\begin{array}{l}\text { The structure } \\
\text { of concept } \\
\text { hierarchy }\end{array}$ & Multiple inheritance & Multiple inheritance & Single inheritance \\
\hline Features & $\begin{array}{l}\text { Bilingual dictionary, collocation dictionary, } \\
\text { corpus, etc. are recorded }\end{array}$ & $\begin{array}{l}\text { Words are divided into } \\
\text { groups by synonym } \\
\text { (synset) }\end{array}$ & $\begin{array}{l}\text { About 12,000 sentence patterns } \\
\text { corresponding to the meaning of verb } \\
\text { concept are recorded. }\end{array}$ \\
\hline
\end{tabular}




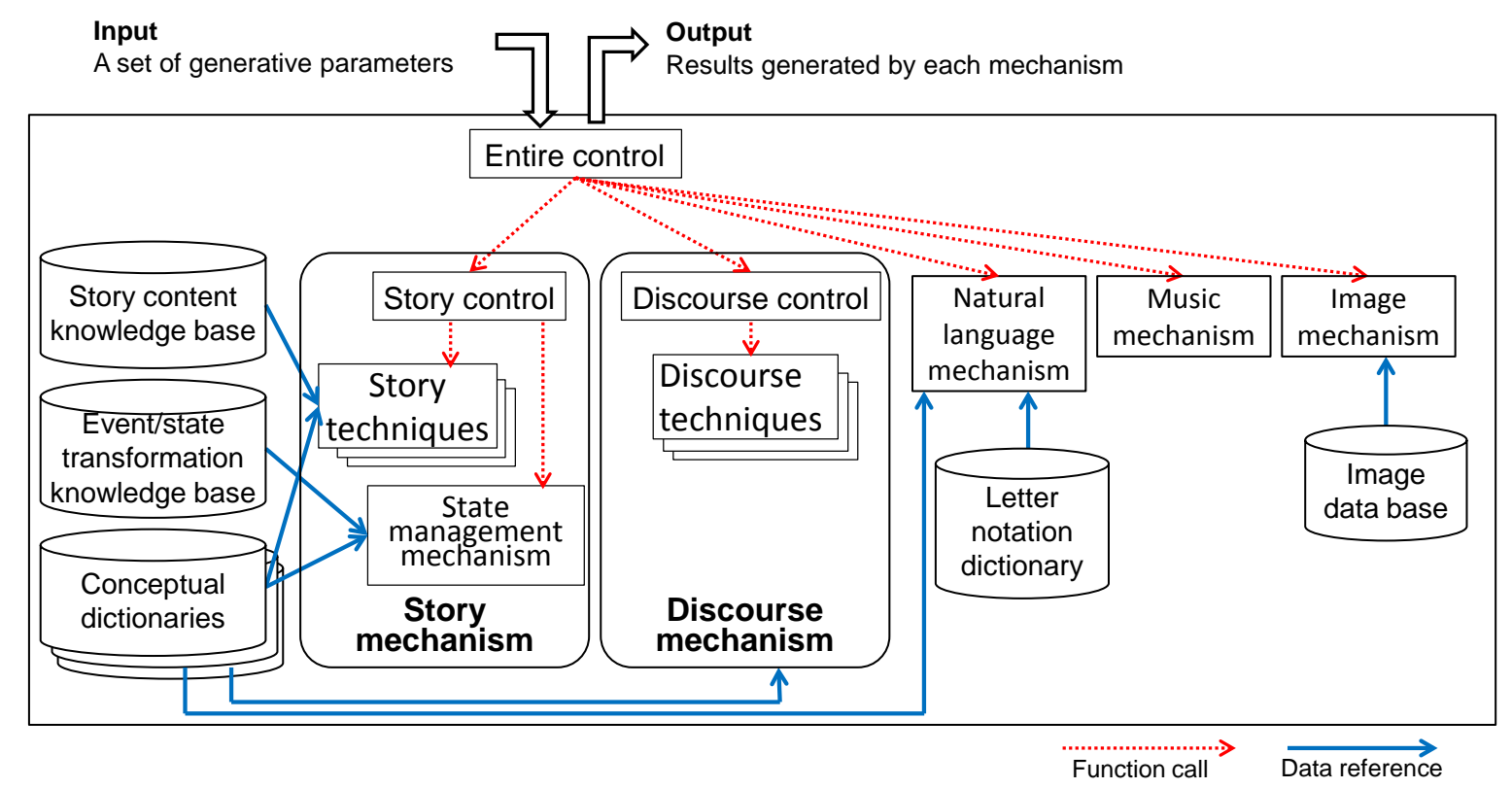

Fig. 1. The Architecture of INGS.

narrative generation. INGS has a knowledge-based architecture including story/discourse/expression phases and several expression media of language/music/image. Next, literary theories or narratology and informatics are integrated through the property of expanded literary theory or information design of narratology. The last 4) means that the processing of each of the phases is executed by the use of a variety of "narrative techniques" which are equal to functions to actually construct various types of narrative structures.

A "story" generated by the story (generation) mechanism means the content of a narrative or a temporal sequence of events. A story is represented by a tree structure consisting of the three basic units of event, relation, and state. An event is described with a conceptual representation form as a frame structure or a case structure including a verb concept and the related noun concepts. A relation semantically combines events or the sub-structures. We have prepared a variety of relations such as "cause-effect”, "script”, etc. An event is principally combined with a preceding state and a subsequent state [26]. A state is equal to the static information in a story, while an event is corresponded to the dynamic information. The chief functions of state processing are to hold the knowledge about a story's world and to manage the coherency of a flow of events. A story structure with events and relations is generated using one or more story techniques and states associating with the events are made according to another mechanism. Propp's folktale theory of a story structure [27] is also used in a part of the story generation phase. This is an trial of our expanded literary theory $[28,29]$.

A “discourse" generated or transformed based on the discourse mechanism means the structural aspect of how to narrate a story. However, a discourse structure to be narrated is distinguished from the surface media to narrate or express it, and accordingly a discourse structure is described with a conceptual representation form. A discourse corresponds to the structure in which a story structure is transformed to the form to be narrated actually. The main part of discourse techniques as a group of narrative techniques is currently defined [30] based on the narrative discourse theory by Genette [31] which is a typical narratological literary theory.

"Expression" means the aspect of narrative representation using various surface media including mainly natural language, such visual media as animation and still image, and music. The surface expression or representation module consists of the following three mechanisms: a natural language mechanism that transforms a story or discourse conceptual representation into a sequence of sentences [32], an image mechanism including an animated movie generation mechanism [33] as a part, and a music mechanism that conducts on the cyclical mutual transformation between conceptual narrative structures and musical structures [16,17]. In the present version of 
INGS, the natural language and image mechanisms are very simple.

Various knowledge elements including conceptual dictionaries are the other very important and essential mechanisms for story generation especially. The knowledge parts in INGS are strongly related to generating narrative contents. The task of narrative generation has both formal and substantial aspects from a point of view. In the story generation mechanism in INGS, for example, story techniques are corresponded to a formal aspect because each of the story techniques is an abstract definition to be completed finally using substantial knowledge. Main three types of knowledge elements are dictionaries, a state-event transformation knowledge base [26], and narrative content knowledge bases. In the present version of INGS, in addition to conceptual dictionaries in this paper, there is a dictionary for surface language generation called language notation (or language presentation) dictionary. The state-event transformation knowledge base is used when the story generation mechanism generates the states according to an event concept generated. The preceding and subsequent states are generated for an event. The last narrative content knowledge bases, ideally, should consist of some types of content knowledge bases associating to narrative content knowledge for story generation, music generation, language generation, etc. In particular, the content knowledge for music generation and language generation respectively has musical motives and fragmental sentences. But the present INGS does not arrive at the stage and a story content knowledge base is partially implementing. Each of story techniques is associated with the story content knowledge which defines the concrete fragmentary sequential structures of events to be used in the corresponding to the story technique. For example, for the "sequence" technique, many content knowledge structures will be able to be defined as a part of the story content knowledge base.

Finally, INGS provides mechanisms for controlling the overall generation level and the level of each of the phases in a narrative generation process. The major function is to decide how to use narrative techniques (for story, discourse, and surface representation including language and music) to direct characteristics of the narrative texts to be generated. A relatively fullfledged control mechanism [30] is currently used in only the discourse phase based on the literary theory by Jauss (a type of reception theories) [34].

\section{Basic Structures and Functions of the Conceptual Dictionaries}

As stated in the previous section, a story and a discourse are mutually represented as a tree structure, and the basic unit is an event or an event concept described as a conceptual representation form. Fig. 2. shows the tree structure of a story and the actual example of an event (“An old person goes to a mountain from a home”). In the story structure, each terminal node in the structure is corresponded to an event, while each intermediate node is a relation for binding the lower nodes. An event is described as a case frame including a verb concept and several noun concepts. A case frame is defined using the following eight basic cases: "agent” (the subject of an action), "counter-agent” (the object of an action and a living thing), "object” (the object of an action and a no living thing), "instrument" (the tool used for an action), "location" (the place of an action), "time" (the pair of the starting time and the ending time of an action), "from" (the starting place of an action) and "to" (the ending place of an action). In addition to these basic cases, nine kinds of optional cases are defined to treat such concepts which are not able to describe using the above cases (Table 2.). As indicated in the table, a part of the optional cases is overlapped to the definition of special cases by Okada [35] and Takeuchi [36]. The first function of the conceptual dictionaries in INGS is to define constituents of an event.

As the scale, the present version of the verb conceptual dictionary has the following elements: 11951 verb concepts (terminal concepts), 36 intermediate concepts and 16079 case frames. The present noun conceptual dictionary has 115769 noun concepts and 5809 intermediate concepts. Both conceptual

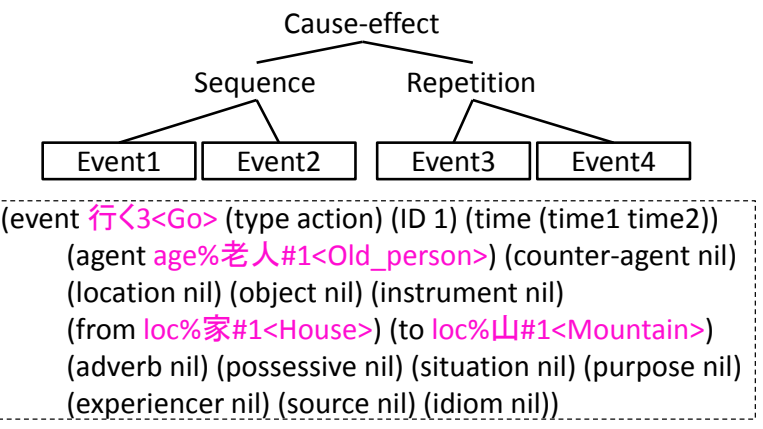

Fig. 2. Examples of Story Structure and an Event. 
Table 2. Optional Cases.

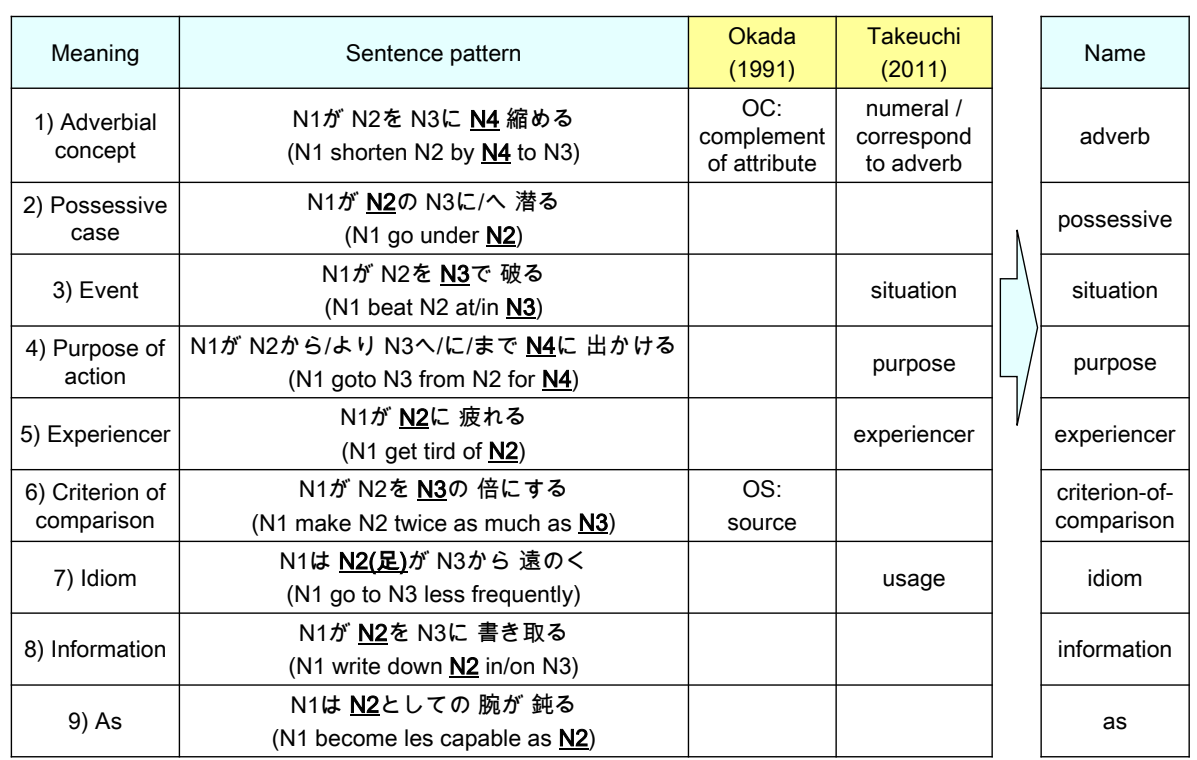

dictionaries respectively have a hierarchical structure from higher concepts to lower concepts according to "is-a" relations with the mechanism of single inheritance. The conceptual dictionaries are all described with Common Lisp as well as the main parts in INGS. Additionally, since intermediate verb concepts in the hierarchy are only 36 in the present version, the elaborated selection of an element is impossible under a hierarchical range in event generation. We are increasing the intermediate hierarchy.

A verb concept defines one or more "case frames" which become template(s) of the event concept and has the description of "constraint condition(s)" (simply "constraint(s)") for limiting the range of each case's value. A constraint is defined using one or more noun concepts existing in the noun conceptual dictionary and means the range that shows an element in a case contained in a verb concept exits within the hierarchy of the noun conceptual dictionary.

If all the noun concepts in an event are within the range of the constraints, this mechanism will enable to generate a natural and possible event like "A knight fight an enemy". However, if the noun concepts out of the range are used, an unnatural and impossible event, like "A knight fight a windmill”, may be generated. As another example, in an event concept, "(eat (agent oldperson) (object rice-ball))"1 ("an old-person eat a riceball”), when "(eat (agent N1) (object N2))" is defined as the case frame corresponding to "eat" and the noun concept of "food" is defined as the constraint of "N2", the noun concept to inserted into "N2" is the subordinate concept of "food" in the hierarchy of the noun conceptual dictionary. Next, a "sentence pattern" is the third information which orders a basic sequence of words based on the case frame to be utilized in the natural language mechanism.

${ }^{1}$ Though the concrete instances are actually used for the "old-person" and "rice-ball", we use the description for the sake of simplicity. 


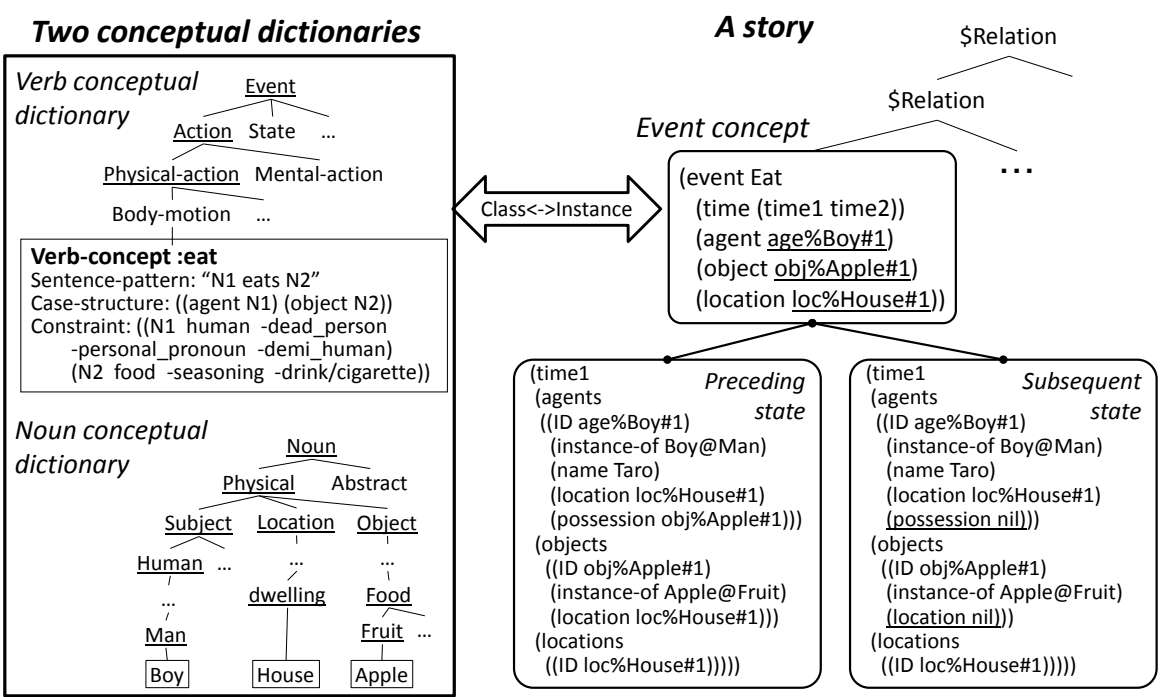

Fig.3. Conceptual Dictionaries and Knowledge Structures of a Story.

Fig. 3. illustrates a relationship between verb and noun conceptual dictionaries and the narrative conceptual representation structures of a story in INGS. The form of a story's conceptual representation is equivalent to a tree structure in which a story is hierarchically described. Two or more events are combined with a relation in a story's tree structure. By a recursive processing, a structure to be combined with a relation sometimes includes two or more partial structures with the other relation(s). Next, at the before and behind of an event in a story, two states of a precedent state and a subsequent state are added to store such background information of the story as characters, places, and objects. In addition to the above function, when an event is generated, INGS automatically makes the corresponding "instance(s)". An instance is the concrete, substantial or actual information of a noun concept. The noun conceptual dictionary has a function to make an instance from each general noun concept described in a case frame according to the description of the attribute frame of the noun concept. But in the present INGS, since the attributes frames is incomplete, the mechanism of instance generation is tentative.

In summary, when the story mechanism generates

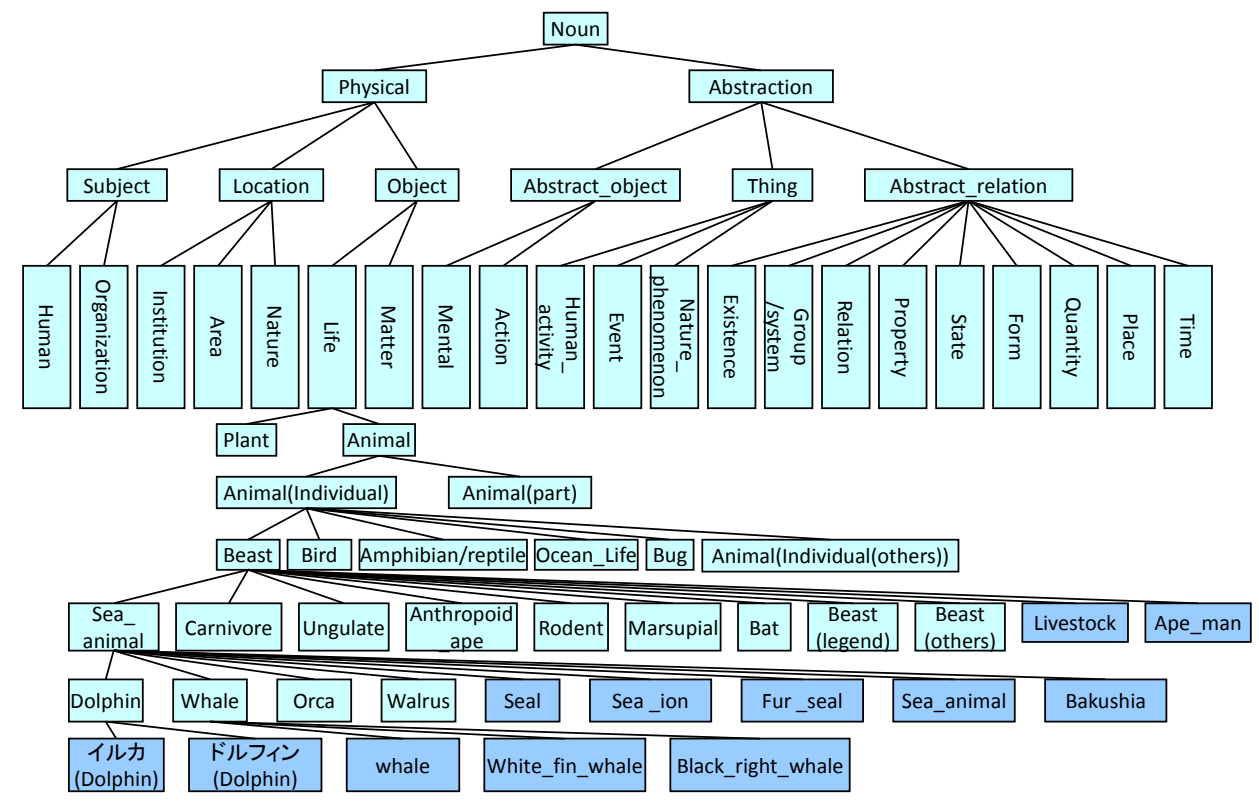

Fig. 4. The Hierarchical Structure of the Noun Conceptual Dictionary. 


\begin{tabular}{|c|c|}
\hline $\begin{array}{l}\text { Description } \\
\text { format (a noun } \\
\text { concept) }\end{array}$ & $\begin{array}{l}\text { (set [noun-concept] } \\
\text { '([noun-concept] } \\
\text { (hierarchy } \\
\text { (depth [number]) } \\
\text { (hype [superordinate-concept]) } \\
\text { (hypo [list of subordinate-concepts]) } \\
\text { (terminal [list of subordinate-terminal-concepts])) } \\
\text { (frame [nil]))) }\end{array}$ \\
\hline $\begin{array}{l}\text { Example (noun } \\
\text { concept } \\
\text { hierarchy) }\end{array}$ & 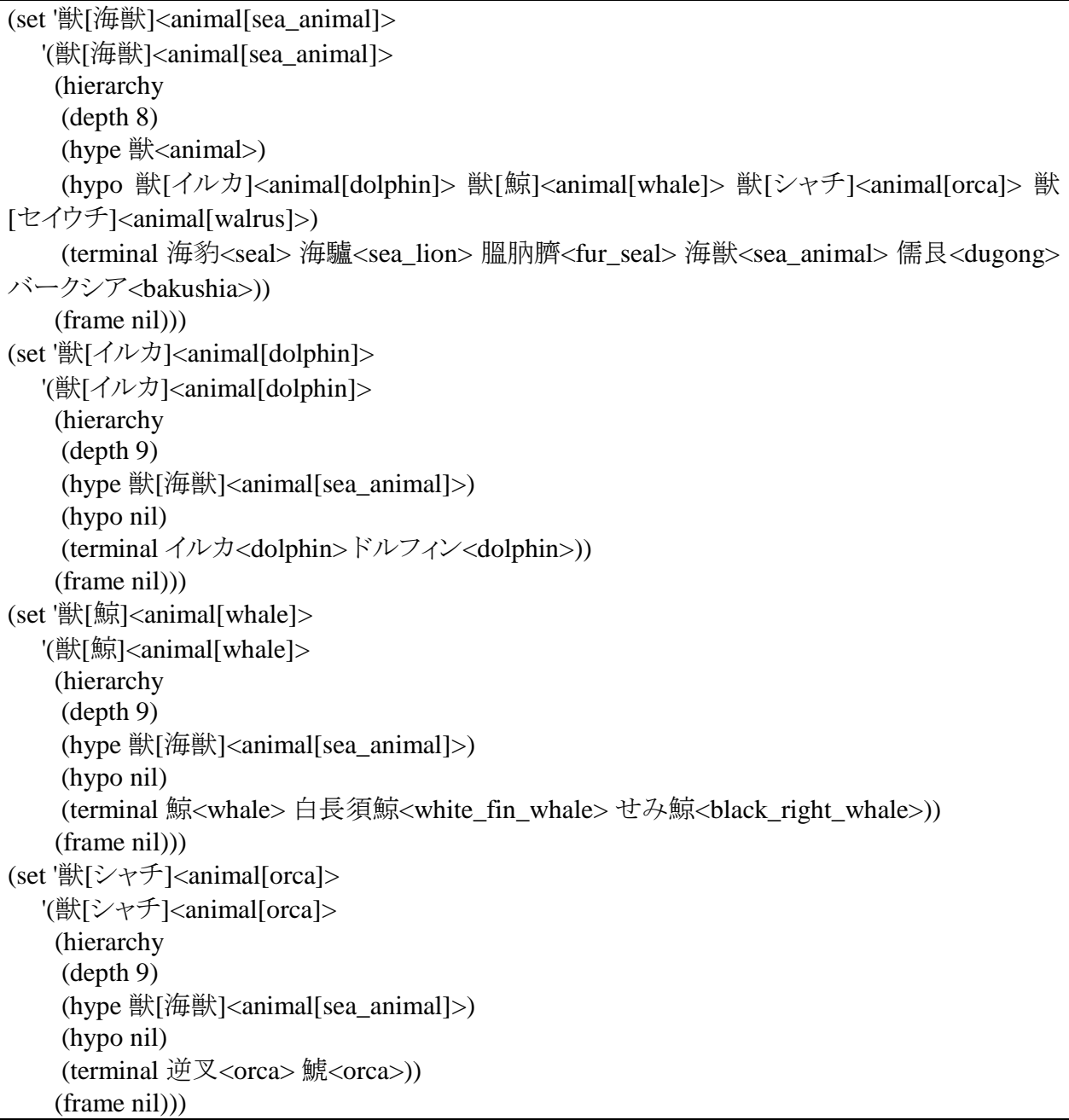 \\
\hline
\end{tabular}

Fig. 5. The Description Format of a Noun Concept and an Example of the Hierarchy.

an event concept, the verb conceptual dictionary provides information of the corresponding case frame and sentence pattern. On the other hand, the noun conceptual dictionary gives one or more noun concepts (general noun concepts and then the instances) to the event according to the constraints in the case frame from the verb conceptual dictionary.

\section{Noun Conceptual Dictionary}

Fig. 4. is the overall structure of the hierarchy of the noun conceptual dictionary ${ }^{2}$. The terminal nodes are equal to the most concrete concepts and the intermediate nodes are corresponded to more abstract concepts to bind the lower parts. In Fig. 5., we show the description format and an actual example and Fig. 6. means the hierarchical structure of the example. Each value of "depth" means the number from the highest level in the

\footnotetext{
${ }^{2}$ Actually, the elements are described by Japanese. In the following description relating to actual conceptual dictionaries, we will show each concept as a pair of the original description by Japanese and the English translation as necessary.
} 


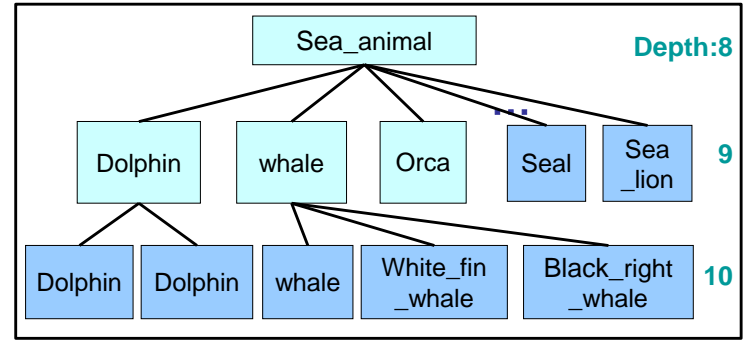

Fig. 6. Hierarchical Structure of the Example in Fig. 5

hierarchy.

As a chief principle for the development of the noun conceptual dictionary, we use the terminal concepts close to language level words as much as possible because of the successive processing between conceptual generation and surface language generation. However, in Japanese, a word that has a meaning ordinarily has several different notations. For example, the word of "tanuki" that means "raccoon dog" has homonyms of “狸” (“tanuki” in Kanji), “タヌキ” (“tanuki” in Katakana), “たぬき” (“tanuki” in Hiragana), etc. The generation process in INGS is divided into conceptual generation and surface representation such as language generation. A noun concept generated using the noun conceptual dictionary is given an actual word representation or one of the notations using a word (or letter) notation dictionary for surface language representation. We have developed the dictionary in which various word notations are described for each noun concepts.

There are two types of important knowledge-based mechanisms relating to the noun conceptual dictionary. Since the development has not finished in the present version of INGS, we refer to only the basic concept and design for the future development here. As mentioned above, each noun concept in an event generated is defined as an instance in INGS. An instance is a proper existence and is often able to be defined using a proper noun. For example, “富士山<mount_Fuji>” is a proper noun. On the other hand, a person's name like “花子 $<$ Hanako $>$ " is a name for a proper and concrete person. Although “富士山<mount_Fuji>” and “花子 $<$ Hanako>” are words, each of the instances needs to have a set of information for the content. Firstly, proper nouns like “富士山<mount_Fuji> are needed to be stored as a part of the noun conceptual dictionary. In particular, each proper noun is linked to the corresponding general noun concept as a proper noun concept. The essential meaning of a "concept" is that it includes an "(noun) attribute frame" which defines the content information. We are currently adding proper noun concepts to the noun conceptual dictionary.
The noun "conceptual” dictionary is necessary to define attribute frames to represent the content of the instance of each noun concept. The advanced processing needs the fulfilling definition although an attribute frame in the present version associating with a noun concept mainly defines only the information about the position in the noun conceptual hierarchy except partialy completed definition and use. For example, we have prepared a tentative list of attributes for narrative characters by an analysis of novels, which includes social, external, internal or psychological characteristics. And we actually use a part of the elements in INGS. Besides, we are beginning the study of the automatic acuisition from various types of texts including novels on the web.

The other main roles of attribute frames are as follows. The first is to generate explanations and descriptions of a noun concept and the group. The mechanism is able to generate explanatory texts of mainly abstract concepts of an instance such as social characteristics of a character and descriptive texts of external concepts such as a character's face and body. These functions are related to both the conceptual structure's construction of an explanation and the natural language generation. The second role is especially relevant to the part of natural language generation by making a noun phrase using an attribute frame for an instance. Figuratively speaking, if events and states in a narrative are equivalent to a trunk in the tree of a narrative, explanations and descriptions using attribute frames are the nuts. As stated above, one of the most significant future tasks of INGS is to systematically build attribute frames combined with the noun conceptual dictionary.

\section{Verb Conceptual Dictionary}

Fig. 7. illustrates the overall hierarchical structure of the verb conceptual dictionary. As shown in the lower part of this figure using the example of “食べる $2<$ eat2>”, a terminal verb concept describes a sentence pattern, one or more case frame(s), and constraint(s).

Next, Fig. 8. shows the description format of a verb-concept and the example. The example includes the following values: (1) A sentence pattern, "N1 eat N2". The "N1" and "N2" are corresponded to the values of "agent" and "counter-agent" or the values of "agent" and "object" in case frames. "N3" is an optional value and is not included in the sentence pattern. (2) Two case frames, "(eat2 (agent N1) (counter-agent N2))" and “(eat2 (agent N1) (object N2))”. (3) Constraints for each of the case frames. For example, the constraint for "N1" in the first case frame is “( 人 < human> - 死人 

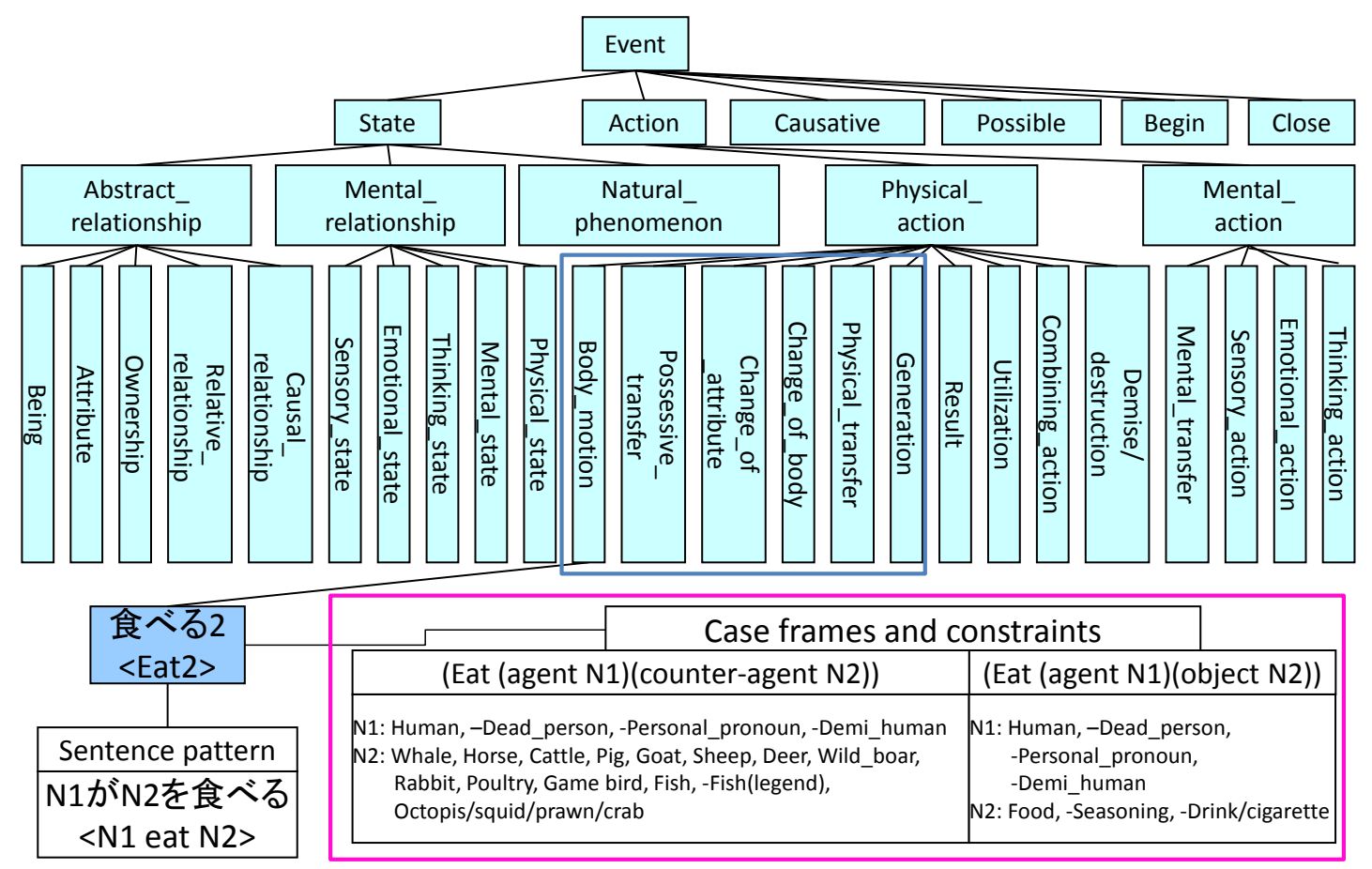

Fig. 7. Hierarchical Structure of the Verb Conceptual Dictionary.

$<$ dead_person $>$ - 人間 $\langle$ 人称 $<$ personal_pronoun $>>$ - 準 人間 $<$ semi_human $>$ )". The first case frame has three lists of constraint for "N1", "N2", and "N3". (4) The list of the super-ordinate concept by "is-a" relation of the concept. In this example, the super-ordinate concept is “( $\mathrm{v}$ 身体動作 $<$ body_motion $>)$ ”.

\subsection{The validity of the constraints}

Adequate case frames of each of noun concepts have been defined based on a sentence pattern and constraints. We show an example of the definition based on a sentence pattern, "N1 eat N2". We simply hypothesize that the constraints of "N1" and "N2" can respectively be "(human, animal)" and "(food, animal)". This means that "N1" takes the subordinate concepts of "human" or "animal" and "N2" takes the subordinate concepts of "food" or "animal". In "N2", "a living thing (animal)" is distinguished from "a nonliving thing (food)" to define the following two types of case frames, "(eat $2^{3}$ (agent N1) (object N2))" and "(eat2 (agent N1) (counter-agent N2))". The constraints of "N1" in the two case frames are commonly "human" and "animal", while the constraints of "N2" are respectively "food" and "animal". As mentioned above, a verb concept sometimes holds some different case frames.

As shown in Table 2., in the present

\footnotetext{
${ }^{3}$ There are many concepts in "eat" and the "eat2" is the one type.
}

implementation, case frames of 266 verb concepts are defined using special nine types of cases instead of basic cases. We explain using the case of "purpose". For a verb concept of "go3", the sentence pattern is "N1 go to N3 from N2 for N4" and the constraint of "N4" is "(abstraction)" which shows the purpose of "go". For instance, "a man goes to a mountain from a house for mowing". If we interpret it as "to", we are not able to distinguish "N4" from "N3" ("(place, field)").

As a relating study, Okada [35] and Takeuchi [36] respectively proposed ten types of cases and more detailed 71 types of ones to represent argument structures of verbs which define the relationship between a verb and nouns in a sentence. Since using the all types may become too long and complex in description, we tentatively use only nine types of cases with "optional" sign.

Additionally, there are various semantic knowledge resources relevant to our research. FrameNet [37] and Japanese FrameNet [38] define the meaning of a word using a semantic frame which means the structured knowledge about a typical scene. VerbNet [39,40] is a hierarchical verb lexicon in which each class is described by semantic predicates, thematic roles, and basic syntactic frames. These resources and the above [36] are advanced semantic knowledge to deal with complex narrative knowledge units. In the modular approach of INGS, each common and relatively large-scaled conceptual dictionary treats the 


\begin{tabular}{|c|c|}
\hline $\begin{array}{l}\text { Description } \\
\text { format (a verb } \\
\text { concept) }\end{array}$ & 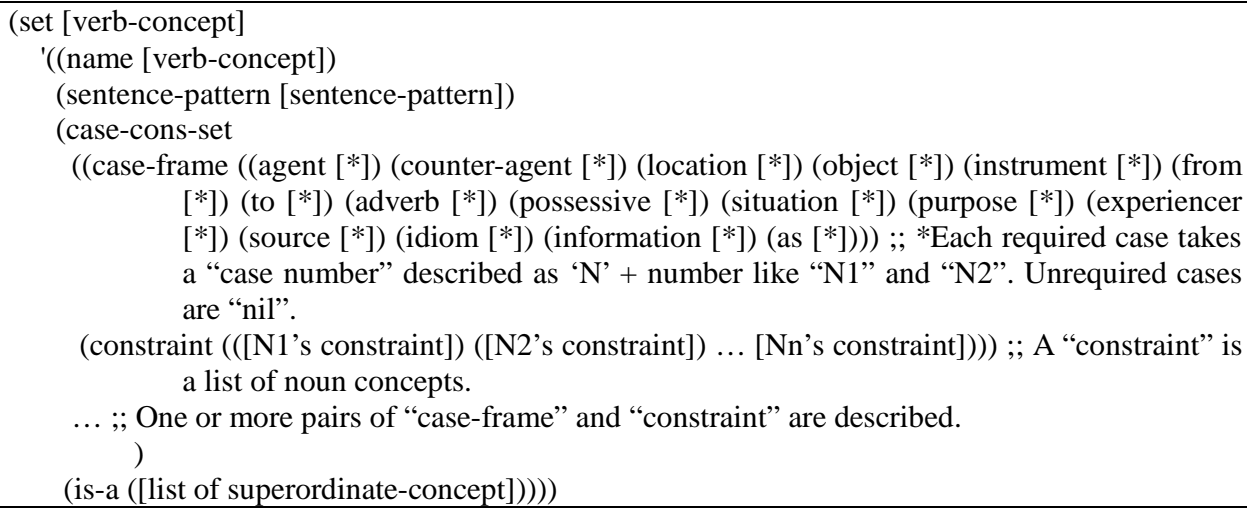 \\
\hline An example & 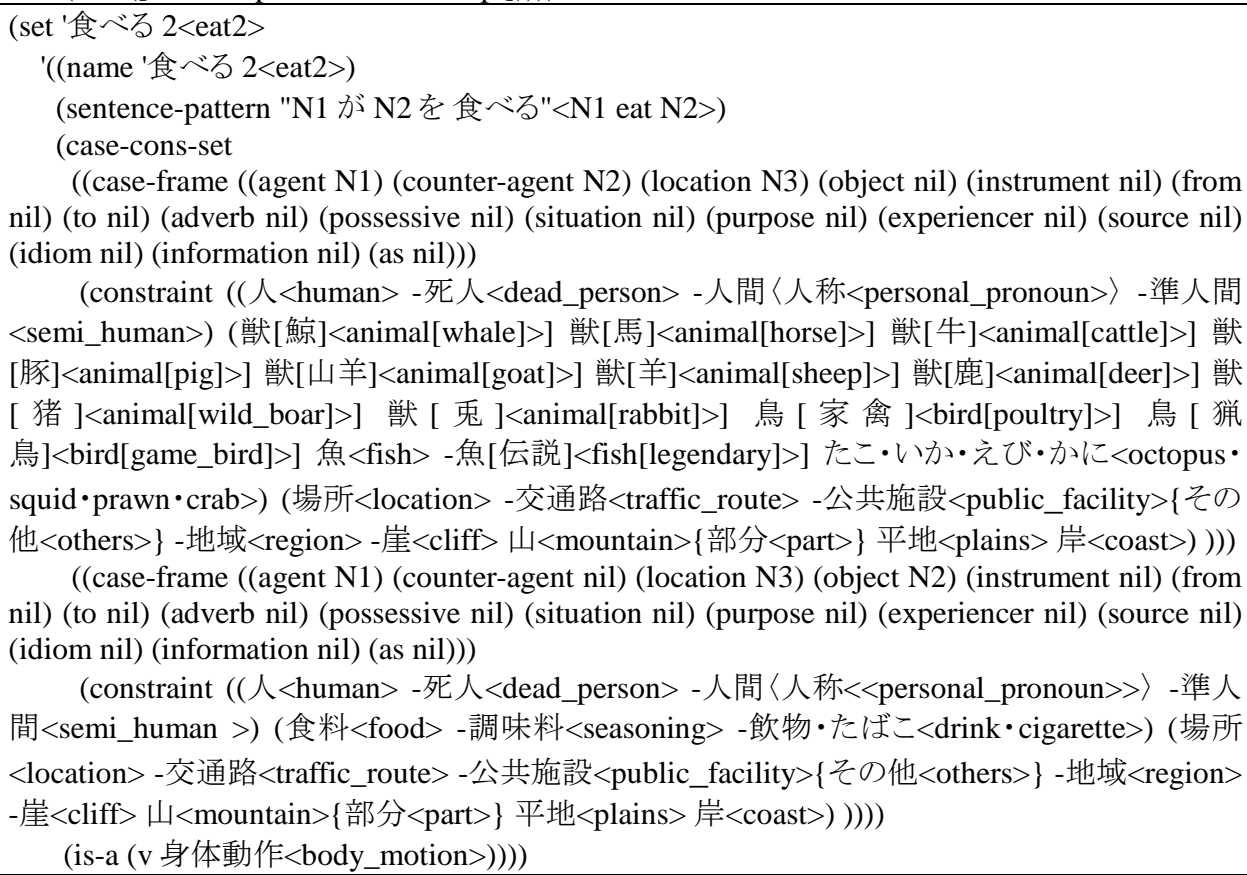 \\
\hline
\end{tabular}

Fig. 8. The Description Format of a Verb Concept and the Example.

semantic knowledge which has a simple structure and another mechanism handles more complex semantic processing. In particular, state-event transformation mechanism [26] generates the preceding and subsequent states from an event using state-event transformation knowledge base which defines transformation rules between an event and the states. The above semantic resources will more closely be connected with the part in INGS.

\subsection{Editing constraints}

A previous evaluation of constraints described in the verb conceptual dictionary indicated a difficult and ambiguous result based on the standard of "actual naturalness". So, we have re-defined the constraints according to the standard of "physical possibility" (in our daily life in common sense) as a basic policy.
Though the method pragmatically has problems, it is relatively clear to define. For example, the purpose and role of constraints described in "A Japanese Lexicon" [22] is originally the conflict resolution for JapaneseEnglish translation. And the definition is too wide to adequately limit the semantic range of a noun concept to be generated in the context of narrative generation. In particular, in case of "eat2", the subordinate concepts of a constraint "food" corresponding to the "N2" include inadequate concepts such as "drink”. One of the primary policies of developing constraints was to avoid such ambiguity and inaccuracy.

We have defined constraints according to the following two ways. The first is based on hyponymy concepts. For example, "furniture" is substituted with "chair" and "bed". The second is based on the partial exclusion of hyponymy concepts. For instance, 
“dead_person” is excluded from "human” using a minus sign such as "-dead_person”. As an actual example, for the constraints of "N3" in "N1 return from N2 to N3", we have defined "(lodging housing area -area(scope) area(human_activity) -land -world foot_of_mountain mountain_pass valley ground island/cape shore -bank farm site point_of_compass edge distance house(body)[housing])”. However, this procedure spent a lot of time.

To improve the problem, we have prepared a lot of "sets" for constraint description. A "set" is a group of constraints to be applied commonly to similar case frames. If a common "set" can be applied to the case frames including "a place where a person can sit down", the "set" can be used to the opposite concepts such as "stand up" too. We give a name for showing a "set" of noun concepts to be used as constraints. A "set" of constraints with a name can be applied to various case frames commonly as a whole. For instance, the "set" of "inhabitable location" is equal to a "set" of constraints, "(lodging housing area -area(scope) area(human_activity) -land -world foot_of_mountain mountain_pass valley ground island/cape shore -bank farm site point_of_compass edge distance house(body)[housing])". The set is commonly used to the constraints in various events, such as "N2" in "N1 move to/into N2". As another example, for "N1 take out N2 from N3", the set of "a person of such age who can take care by herself or himself" is applied to "N1" and the set of "transportable object" is applied to "N2". In the first development stage of the verb conceptual dictionary in INGS, we have defined the constraints for only agent, counter-agent and, object because they are minimal information of making a sentence. And then, on the basis, we have added the constraints for location cases [41]. Finally, 38 sets have been defined and used in the present version of the verb conceptual dictionary.

\subsection{Defamiliarization using constraints}

This part is out of the direct theme of the paper. In the combination with the field of narrative generation, the possibility of a variety of novel and difficult applications emerges in the use of the conceptual dictionaries in INGS. "Defamiliarization" using the constraints is an application [42]. Fantastical events or physically impossible events often appear in narratives. Our narrative generation project or INGS aims to be able to flexibly generate from more realistic narratives or event sequences to more fantastical ones. Realistic narratives and fantastical narratives respectively need different ranges of constraints. The proposed conceptual dictionaries in this paper provide a foundation of the function of defamiliarization. The above application adjusts the semantic constraints of concepts in a single event in a short advertising scenario generated using a part of INGS. The function has been implementing by using "defamiliarization" techniques. The idea was originally acquired from a rhetorical analysis of actual television commercial films. The analysis defined three types of standard rhetoric to generate ordinary events and nine types of irregular rhetoric to generate extraordinary events. We call these twelve types of rhetoric "product introduction techniques". In advertising narratives, events with a product are generated through the techniques. The standard or ordinary techniques draw the process of "manufacturing”, "purchasing”, and "usage” of a product, while the extraordinary techniques conduct on a kind of deviance processing by the defamiliarization techniques for “actor's action”, “product's state”, "background or place”, "actor himself (herself)", and so on. Defamiliarization means a literary idea [43] that changes a familiar object into unfamiliar one to reinforce the impression. The impression of a familiar product can be reinforced by applying of defamiliarization techniques to the objects and agents or actors. The technological idea will be able to generalize to one of the basic concepts of INGS, which we have called it the concept of "norm and deviation", more than advertising narrative processing. The basis of the mechanism is the conceptual dictionaries, especially the constraints.

\section{Verification and Consideration}

This section tentatively verifies and considers the current achievement of the conceptual dictionaries from some view points for the future reform and expansion.

\subsection{The validity of the constraints}

For the verb concepts to which a constraint "set" is applied, we verified the validity and the classification of noun concepts associating to the constraints. The criterion is whether physically possible events can be generated under the limitation of each constraint. Such event as "A salesgirl divorces her husband" is possible and such event as "A spokesman is sailing on the paper" is impossible.

We implemented an experimental event generation program that selects a noun concept for each case in the case frame at random according to the constraint. Using the program, 20 events were generated by 100 case frames selected at random from 1158 case frames in which all constraints were set. Three evaluators checked the generated total 2000 events dependent on the 
standard of "possible/impossible". We decided that the constraints in the case frame were relatively adequate if $14 / 20$ or more events were "physically possible". When the results were different by evaluators, we employed the biggest number.

As a result, success (relatively adequate) was 70 and failure (inadequate) was 30. For 12 in the 30 failures, we modified them by using another set of constraints. For example, in the case frame of "N1 collect N2 from N3", we may replace the set of "N2:object" from "goods, status and notion to be able to send" to "goods to be able to send". For 15 in the failures, we modified the constraints themselves. For instance, in the case of "N1 sail N2", we changed the constraint of "N2:location" to "(river waterway lake sea)" from "(river waterway lake sea sky)". The latter was based on the set, "the place to be able to pass with ship and aircraft”. The other two failures were simple description errors of case frame. Remaining one problem was the case that the verb concept itself is impossible such as "N1 cast a spell on N2".

By using the above standard of evaluation, the difference of feeling by evaluators and the ambiguity of evaluation diminished more than previous criterion, natural/unnatural. And it contributed to the simplicity of system development. However, there is also the difficulty that we cannot eliminate possible but ordinarily unnatural events. Because it is hard to absorb such kind of contextual knowledge into the conceptual dictionaries, the solving needs to be given in the relation with the above-mentioned defamiliarization rhetoric. Our basic policy is that the conceptual dictionaries provide relatively simple and standard conceptual knowledge, while a variety of narrative knowledge such as defamiliarization operates and adjusts more complex and advanced literary or artistic rhetorical techniques. In INGS, the latter knowledge can be stored and organized into various knowledge bases including story contents knowledge base and various techniques such as story techniques and narrative discourse techniques utilize and operate the knowledge to complicate and elaborate the narrative generation.

\subsection{The procedure of defamiliarization processing}

This section presents a processing flow of the defamiliarization concept applying constraints based on the simplest way [42]. First, the user selects an advertising product name as an object concept from a products list prepared by the developer and the number (1-3) to specify a type of "standard techniques" that means the techniques to make an ordinary event in an advertising context. Based on the information, the system generates an ordinary event by acquiring a verb concept and the noun concepts within the selected standard technique for the product. These verb and noun concepts are prepared for each product. Next, the user inputs the number of an irregular (extraordinary or defamiliarization) technique and the system rewrites the ordinary event to an extraordinary event by applying the corresponding rhetorical technique to one or more of the elements in the former event. The conceptual dictionaries are used for applying the extraordinary rhetoric. A defamiloarization technique replaces an original noun concept in a target event with another noun concept in the different category by changing the reference space in the noun conceptual dictionary.

The mechanism, for example, generates an event with the product of "car" according to the "defamiliarization of location" technique. First, the mechanism generates an event such as "(drive (agent woman) (instrument car) (location plateau))" by using the noun concept of "car" included in the constraint. Next, the mechanism replaces the noun concept corresponding to the location case with another concept outside the constraint using the defamiliarization technique to generate a new event at a strange place such as "(drive (agent woman) (instrument car) (location seabed))". The verb conceptual dictionary principally defines a standard semantic range for each noun concept in an event from the viewpoint of actual possibility or physical possibility. In contrast, extraordinary or irregular events are constructed by several types of defamiliarization techniques dependent on a kind of constraint relaxation mechanism. We have introduced the defamiliarization mechanism into the story generation mechanism in INGS as a group of story techniques. But, the result had not been efficiently effective because of the random or non-strategic application of the defamiliarization techniques and so on. More systematic and elaborating approach will be necessary in the future implementation.

\section{Conclusions}

In this paper, we have presented two dictionaries for Integrated Narrative Generation System (INGS), verb conceptual dictionary and noun conceptual dictionary. Each of the dictionaries has a common conceptual hierarchy of single inheritance by is-a relations. Each terminal concept in the verb conceptual dictionary describes one or more case frame(s), the main element's constraints, and a sentence pattern. The basic description of each terminal concept in the noun conceptual dictionary associates the concept to the other noun concept. Although a noun concept needs an 
attribute frame, the description is incomplete in the present version. The purpose of this research is not developing general conceptual dictionaries, but building them as a mechanism in narrative generation. In particular, we have actually and organically combined the two conceptual dictionaries with INGS. The specific form of an event that is a basic unit in a narrative is given by a case frame and the constraints in the verb conceptual dictionary, and the content knowledge is provided according to the noun conceptual dictionaries. We have presented the basic mechanism based on the cooperation of two types of conceptual dictionaries in INGS. The research has various problems and future issues. One issue to be considered is about the validity of constraints. Since narratives have a width from realistic style to fantastical style, plural standards should be dealt with in a narrative generation mechanism. We have presented an idea by adjusting and operating constraints using the rhetoric of defamiliarization. As the other future issues, we have referred to expanding the hierarchy of the verb conceptual dictionary and developing the parts of proper nouns and attribute frames in the noun conceptual dictionary.

\section{References}

1. R. C. Schank and R.P. Abelson, Scripts, Plans, Goals, and Understanding: An Inquiry into Human Knowledge Structures (Lawrence Erlbaum, NJ, 1977).

2. J. R. Meehan, The Metanovel: Writing Stories by Computer (Garland Publishing, NY, 1980).

3. E. T. Mueller, Daydreaming in Humans and Machines (Ablex, NJ, 1990).

4. S. R. Turner, The Creative Process: A Computer Model of Storytelling and Creativity (Lawrence Erlbaum, NJ, 1994).

5. N. Okada and T. Endo, Story generation based on dynamics of the mind, Computational Intelligence. 8(1) (1992) 123-160.

6. L. Pemberton, A modular approach to story generation, in Proceedings of the Fourth Conference on European Chapter of the Association for Computational Linguistics (1989) 217-224.

7. R. R. Lang, A declarative model for simple narratives, in Narrative Intelligence: Papers from the 1999 AAAI Fall Symposium, Technical Report FS-99-01 (1999) 134-141.

8. S. Bringsjord and D. A. Ferrucci, Artificial Intelligence and Literary Creativity: Inside the Mind of BRUTUS, a Storytelling Machine (Lawrence Erlbaum, NJ, 1999).

9. J. Bates, Virtual reality, art, and entertainment, The Journal of Teleoperators and Virtual Environment. 1(1) (1991) 133-138.

10. N. Montfort, Generating Narrative Variation in Interactive Fiction (A Dissertation in Computer and Information Science, University of Pennsylvania, 2007).
11. T. Ogata, and A. Kanai, An Introduction to Informatics of Narratology: Towards the Thoughts and Technologies of Narrative Generation (Gakubun-sha, Tokyo, 2010).

12. T. Akimoto and T. Ogata, (2012) Macro structure and basic methods in the integrated narrative generation system by introducing narratological knowledge, in Proceedings of 11th IEEE International Conference on Cognitive Informatics and Cognitive Computing (2012) 253-262.

13. T. Akimoto and T. Ogata, (2014). An information design of narratology: the use of three literary theories in a narrative generation system, The International Journal of Visual Design (2014) in press.

14. T. Ogata, Expanded literary theory: cognitive/computational expansion of literary theories and narratology, in Proceedings of 17th Congress of the International Association of Empirical Aesthetics (2002) 163-166.

15. T. Ogata, S. Imabuchi and T. Akimoto, Narratology and narrative generation: expanded literary theory and the integration as a narrative generation system (2), in Proceedings of the 5th Augmented Human International Conference (2014) P-10.

16. T. Akimoto and T. Ogata, The expansion of paths in the mutual transformation mechanism of music and narrative, International Journal of Cognitive Informatics and Natural Intelligence. 7(4) (2013) 44-63.

17. T. Akimoto and T. Ogata, Circulative narrative generation based on the mutual transformation between narrative conceptual structures and music in the integrated narrative generation system, in Proceedings of International Conference on Artificial Life and Robotics (2014) 36-39.

18. K. Oishi and T. Ogata, Towards the development of conceptual dictionary for narrative generation system, in 7th International Conference on Natural Language Processing and Knowledge Engineering (2011) 351-358.

19. K. Oishi and T. Ogata, The development of conceptual dictionary for narrative generation system: The structure and functions. in Proceedings of the 4th IEEE International Conference on Digital Game and Intelligent Toy Enhanced Learning (2012) 168-170.

20. K. Oishi, Y. Kurisawa, M. Kamada, I. Fukuda, T. Akimoto and T. Ogata, Building conceptual dictionary for providing common knowledge in the integrated narrative generation system. in Proceedings of the 34th Annual Conference of the Cognitive Science Society (2012) 2126-2131.

21. N. McIntyre and M. Lapata, Learning to tell tales: a datadriven approach to story generation, in Proceedings of the 47th Annual Meeting of the ACL and the 4th IJCNLP of the AFNLP (2009) 217-225.

22. S. Ikehara, M. Miyazaki, S. Shirai, A. Yokoo, H. Nakaiwa, K. Ogura, Y. Ooyama and Y. Hayashi, GoiTaikei: A Japanese Lexicon, CDROM (Iwanami-Shoten, Tokyo, 1999). 
23. F. Bond, H. Isahara, S. Fujita, K. Uchimoto, T. Kuribayashi and K. Kanzaki, Enhancing the Japanese WordNet, in The 7th Workshop on Asian Language Resources, ACL-IJCNLP 2009 (2009) 1-8.

24. EDR Electronic Dictionary. http://www2.nict.outpromotion/techtransfer/EDR/J_index.html

25. Japanese WordNet. http://nopwww.nict.go.jp/wn-ja

26. K. Onodera and T. Ogata, Sequence generation based on mutual relationship between state and action: as a mechanism in narrative generation system, in Proceedings of the $4^{\text {th }}$ DIGITEL (2012) 159-161.

27. V. Y. Propp, Morphology of the Folktale, 2nd Ed. Transl. L. Scott. (TX: University of Texas Press, TX, 1968)

28. S. Imabuchi and T. Ogata, A story generation system based on Propp theory: as a mechanism in an integrated narrative generation system, in Lecture Notes in Artificial Intelligence 7614, eds. H. Isahara and K. Kanzaki 312321.

29. S. Imabuchi and T. Ogata, Methods for generalizing the Propp-based story generation mechanism, in Lecture Notes in Computer Science 8210, eds. T. Yoshida, G. Kou, A. Skowron, J. Cao, H. Hacid and N. Zhong (Springer-Verlag, NY, 2013) 333-344.

30. T. Akimoto and T. Ogata, Towards a discourse mechanism in narrative generation system: proposal of a system introducing narrative discourse theory and reception theory. Cognitive Studies. 20(4) (2013) 396420.

31. G. Genette, Discours du Récit, Essai de Méthode, Figures III. (Seuil, Paris, 1972). Transl. J. E. Lewin, Narrative Discourse: An Essay in Method. (Cornell University Press, NY, 1980).

32. H. Sato, K. Kobayashi, T. Yasuda and T. Ogata, A Simple Generation of Single Sentences and Complex Sentences in a Narrative Generation System, in Proceedings of the Japanese Information Processing Society Annual Conference (4) (2008) 4ZH-6 (4 803804).

33. T. Ogata, Towards image generation in a narrative generation system, in Theories and Practices of Image Editing, eds. A. Kanai and Y. Niwa (Hosei University Press, Tokyo, 2008) 165-235.

34. H. R. Jauss, Literaturgeschichte Als Provokation. (Suhrkamp Verlag, Frankfurt am Main, 1970). Transl. T. Bahti, Literary history as a challenge to literary theory, in Toward an Aesthetic of Reception (University of Minnesota Press, MN, 1982) 3-45.

35. N. Okada, Representation and Storage of Word Concepts (The Institute of Electronics, Information and Communication Engineers, Tokyo, 1991).

36. K. Takeuchi, Construction of thesaurus of predicateargument structure for Japanese verbs. in Proceedings of the $25^{\text {th }}$ JSAI (2011) 3H2-OS3-5.

37. C. J. Fillmore and C. Baker, A frames approach to semantic analysis, in The Oxford Handbook of Linguistic Analysis, eds. B. Heine and H. Narrog (Oxford University Press, 2010) 313-339.
38. K. H. Ohara, Lexicon, grammar, and multilinguality in the Japanese FrameNet, in Proceedings of the $6^{\text {th }}$ International Conference on Language Resources and Evaluation (2008) 3264-3268.

39. K. Kipper-Schuler, G. T. Dang and M. Palmer, Classbased construction of a verb lexicon, in Proceedings of AAAI-2000 (2000) 691-696.

40. K. Kipper-Schuler, VerbNet: A Broad-Coverage, Comprehensive Verb Lexicon (Ph.D. thesis, Computer and Information Science Department., University of Pennsylvania, 2005).

41. K. Sugita, T. Akimoto, J. Ono and T. Ogata, The constraint definition of location cases to the verb conceptual dictionary in an integrated narrative generation system, in Proceedings of the Japanese Language Processing Society Annual Conference (2014) 221-223.

42. Y. Zhang, J. Ono and T. Ogata, Single event and scenario generation based on advertising rhetorical techniques using the conceptual dictionary in narrative generation system, in Proceedings of the $4^{\text {th }}$ DIGITEL (2012) 162164.

43. V. Shklovsky, Transl. B. Sher, Theory of Prose (Dalkey Archive Press, IL, 1990). 